\title{
Teledetección no es Tele-adivinación: La importancia de las causas en Viticultura de Precisión
}

\section{Teledetection is not Tele-divination: The importance of the causes in Precision Viticulture}

\author{
Vicente D. Gómez-Miguel ${ }^{1, a}$, Vicente Sotés ${ }^{1}$, y María L. González-SanJosé2 \\ ${ }^{1}$ Departamento de Producción Agraria, Universidad Politécnica de Madrid (UPM), España \\ ${ }^{2}$ Department of Biotechnology and Food, Science, Universidad de Burgos (UBU), España
}

\begin{abstract}
Resumen. La cartografía realizada a partir de la información procedente de sensores remotos es un producto de actualidad que se ofrece con éxito gracias principalmente a su atractiva presentación y a su bajo coste. La información se obtiene mediante sensores instalados en satélites, aviones y otras aeronaves a alta, media o baja altura, tripuladas o no y en vehículos terrestres autopropulsados o no. Los sensores y elementos asociados son capaces de procesar la radiación de gran parte del espectro electromagnético y convertirla en imágenes que representan la emisión de una determinada zona geográfica. A pesar de que el procedimiento puede llegar a ser muy sofisticado, el resultado que se proporciona al viticultor con mayor o menor detalle (resolución) o más o menos elaborado, es prácticamente siempre el mismo: uno o varios mapas en los que se recoge la respuesta obtenida por el sensor de lo que hay en la parcela en el preciso momento en el que pasa el vehículo en el que se ha instalado. Por lo tanto, este mapa representa la respuesta del estado "electromagnético" de una parcela en un momento determinado, sin embargo, las causas de la variabilidad que se aprecia en el propio mapa se desconocen. Estas causas son muy complejas y se relacionan principalmente con la variabilidad de los elementos del clima, la geología, el relieve, el suelo, la planta y el momento de realización del proceso. Factores todos ellos de gran importancia en la configuración del terroir y que influyen en la calidad del producto. En este trabajo tratamos del alcance real de la teledetección y de la importancia de la cartografía de las causas que inciden en la heterogeneidad de la parcela/explotación para un uso adecuado en Viticultura de Precisión, todo ello dentro de un modelo general de producción vitícola para la obtención de vinos de calidad.
\end{abstract}

\begin{abstract}
The cartography that has been carried out from the information coming from remote sensing is a current issue that is successfully offered mainly due to its attractive presentation and to its low cost. The information is obtained by sensors installed in satellite in/on satellites, planes and other aircraft at high, medium or low altitude - manned or unmanned- and in land vehicles - self-propelled or non-propelled. The sensors and associated elements are able to process the radiation of a great deal of the electromagnetic spectrum and transform it into images representing the emission of a certain geographical area. In spite of the procedure can be really sophisticated, the result given to the winegrower, with more or less detail (resolution) or more or less elaborated, is practically always the same: one or various maps with the answer, obtained by the sensor, of the elements in the field in the precise moment that the vehicle, where it is installed, passes. Therefore, this map represents the response of the "electromagnetic" state of a certain moment however the causes of the variability observed in the same map are unknown. These causes are very complex and they are in relationship mainly with the variability of the elements of climate, geology, relief, soil, plant and the moment of the process execution. All these factors are of a great importance in the configuration of the terroir and they influence the product quality. In this work, we deal with the real range of teledetection (remote sensing) and the importance of the cartography of the causes that affect the heterogeneity of the field / exploitation in order to get a suitable use for the Precision Viticulture. The whole of it is inside a general model of vine-growing production to obtain quality wines.
\end{abstract}

\section{Introducción}

La cartografía realizada a partir de la información procedente de sensores remotos (remote sensing) es un producto de actualidad que se ofrece con éxito a los viticultores gracias principalmente a su atractiva

a e-mail: vicente.gomez@upm.es presentación y a su bajo coste. La oferta disponible en el mercado promete al usuario un número importante de elementos cartografiados mediante teledetección que son simplemente imposibles de obtener: teledetección no es tele-adivinación.

La información se obtiene mediante sensores instalados en satélites, aviones y otras aeronaves a alta, media o baja altura, tripuladas o no y en vehículos terrestres

(C) The Authors, published by EDP Sciences. This is an Open Access article distributed under the terms of the Creative Commons Attribution License 4.0 (http://creativecommons.org/licenses/by/4.0/). 
autopropulsados o no. Los sensores y elementos asociados son capaces de procesar la radiación de gran parte del espectro electromagnético y convertirla en imágenes que representan la emisión de una determinada zona geográfica. Las más usuales se corresponden con el espectro visible, el infrarrojo y el radar.

A pesar de que el procedimiento puede llegar a ser muy sofisticado, el resultado que se proporciona al viticultor con mayor o menor detalle (resolución) o más o menos elaborado, es prácticamente siempre el mismo: uno o varios mapas en los que se recoge la respuesta obtenida por el sensor de los elementos que hay en la parcela sólo en el preciso momento en el que pasa el vehículo en el que este se ha instalado. Por lo tanto, este mapa representa la respuesta del estado "electromagnético" de una parcela en un momento determinado, sin embargo, las causas de la variabilidad que se aprecia en el propio mapa se desconocen.

En este trabajo tratamos del alcance real de la teledetección y de la importancia de la cartografía de las causas que inciden en la heterogeneidad de la parcela/explotación para un uso adecuado, tanto en Manejo Convencional o Tradicional (MC) como en Viticultura de Precisión (VP), todo ello dentro de un modelo general de producción vitícola para la obtención de vinos de calidad.

\section{Causas de la Variabilidad}

Las causas de la variabilidad espacial de los viñedos son muy complejas y se relacionan principalmente con la de los factores naturales como el clima (insolación, temperatura, precipitación,...), la geología (litología, mineralogía,...), el relieve (altitud, pendiente, orientación, exposición...) y el suelo (propiedades físicas, químicas, biológicas, mineralógicas,...); de los factores biológicos (patrón, cultivar, biodiversidad intrínseca, estado fisiológico, plagas, enfermedades,...); y de los factores agronómicos (preparación, protección y laboreo del suelo, fertilización, conducción, sanidad, riego...). Estos factores pueden ser modificados por el hombre mediante lo que se describe frecuentemente con los términos gestión o manejo. Factores todos ellos de gran importancia en la configuración del terroir ambiental y que influyen en la calidad del producto [1].

Estos factores, los procesos enológicos, los elementos socioeconómicos y los relacionados con la presentación y el marketing, e incluso, la tradición son los constituyentes del terroir y la metodología de su estudio constituye la Zonificación de los Terroir (Terroir Zoning), o mejor, la Zonificación Integrada del Terroir (ZIT) [2].

A pesar de su importancia, a la hora de establecer el terroir como unidad de análisis (y de manejo) nos encontramos con una barrera que la mayoría de las veces resulta insalvable: la falta de datos enológicos, de mercado y socioeconómicos georreferenciados, es decir, que puedan ser plasmados en un mapa, en definitiva, que sean cartografiables. Este es el motivo principal por el que se utiliza preferentemente el primer grupo de factores para la elaboración del mapa de las causas: el Mapa de Variabilidad del Terroir (MVT) o sus sinonimias: mapa de variabilidad espacial del viñedo, mapa de los terroir o mapa de zonificación del terroir [3].

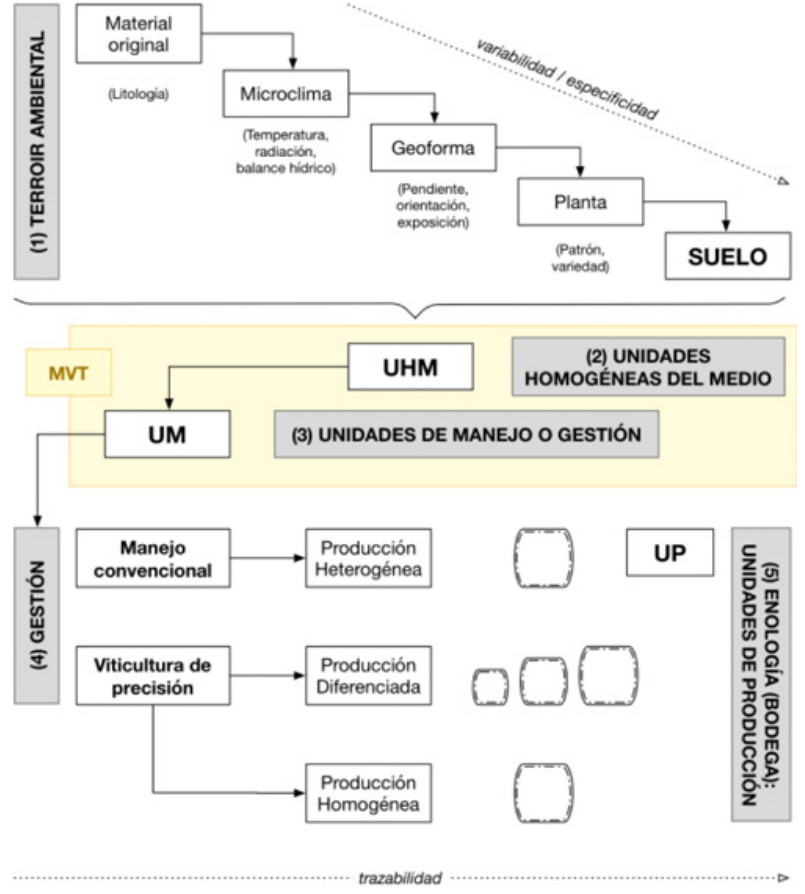

Figura 1. Esquema del modelo de producción vitícola para la obtención de vinos de calidad (para explicación, ver texto).

\section{Importancia de la causas en el sistema de gestión del viñedo}

En la Fig. 1 se incluye un esquema del modelo de producción vitícola para la obtención de vinos de calidad.

El MVT se sitúa como una necesidad entre el Terroir (ambiental) y la gestión del viñedo y está constituido por el mapa de las Unidades Homogéneas del Medio (en adelante, UHM) y el de las Unidades de Manejo o Gestión (en adelante, UM).

El modelo general de producción vitícola se realiza en cinco etapas que comienzan con la identificación, descripción, caracterización y cartografía de los elementos que constituyen el terroir ambiental (1), todos en una escala de detalle comparable. A continuación se crean las UHM (2) y a partir de ellas, se diseñan las UM (3) que permiten la toma de decisiones sobre el modelo de gestión (4) ya sea como MC o como VP. Finalmente, la relación con las Unidades de Producción (por ejemplo: uno o varios depósitos) localizadas en bodega se realiza a través de estas UM y en función del modelo de gestión previamente definido (5). Las tres fortalezas del sistema son el manejo diferencial (cada terroir se maneja de forma particularizada), el aprovechamiento de la especificidad (cada terroir produce un vino determinado) y la trazabilidad (cada terroir se relaciona con su producto de procedencia).

\section{Cartografía de las causas: el mapa de Variabilidad del terroir}

La variabilidad del medio que soporta la viña (variabilidad espacial del viñedo o del terroir) y las dificultades que esta conlleva para una gestión adecuada están en el origen de la heterogeneidad del producto (uva) y en definitiva de la calidad del vino. 
¿Es posible medir, caracterizar, cuantificar y cartografiar la variabilidad espacial del viñedo? ¿Existen técnicas disponibles para ello? ¿Cuáles son esas técnicas?

Un MVT responde a estas cuestiones y representa la caracterización, cuantificación y cartografía de los sectores de la parcela que son similares y su separación de aquellos que son diferentes, lo que permite un tratamiento deferencial.

¿Por qué es importante el tratamiento diferencial de cada sector de cada parcela/explotación? Lo es, tanto si optamos por un objetivo simple o anual como por un objetivo ambicioso o a medio plazo o permanente.

Nos referimos a un objetivo simple cuando se plantea cada año y está relacionado con una propiedad concreta de un determinado factor o un elemento concreto y previamente detectado y seleccionado y sin interferencias de los demás que puede ser cartografiado utilizando un tipo de recursos adecuado. En Viticultura este tipo de objetivos suele estar relacionado con la mejora anual del producto (en cuanto a calidad y/o producción) y los elementos de que depende y que se pretenden corregir frecuentemente se detectan indirectamente y son, por ejemplo, carencias y toxicidades del cultivo, plagas y enfermedades, vigor, estrés hídrico, exceso o falta de humedad, factores de calidad puntual, maduración y vendimia, clorosis, salinidad, etc. En definitiva, se incide en la detección de la diferenciación del sector (por ejemplo, cambio en el vigor) no de sus causas.

Sin embargo, en la Viticultura moderna es frecuente el planteamiento de un objetivo ambicioso que perdure en el tiempo, a futuro, para lo que es necesario conseguir la optimización y homogeneización estable del producto. Para alcanzar este objetivo es imprescindible el MVT y un sistema de gestión que, como la VP, permita actuar de forma independiente en cada propiedad de cada factor y en cada sector de cada parcela.

Se trata por lo tanto de la identificación de las causas de la variabilidad espacial intraparcela a través de las interrelaciones entre las diferentes propiedades plasmadas en su cartografía para su utilización en VP como sistema de gestión o manejo de la explotación. Dicho de otra manera, se pretende solucionar problemas inmediatos dependientes de propiedades específicas e interrelacionadas y hacer a corto o medio plazo (futuro), mediante la gestión llevada a cabo con VP, que los sectores de la parcela sean lo más homogéneos posible y que de esta manera también el producto lo sea.

El MVT permite y facilita determinadas actuaciones que se llevan a cabo en la gestión del viñedo y que no se podrían realizar si este no existiera (Tabla 1). Estas actuaciones están relacionadas, como no podía ser menos, con la VP, sin embargo no hemos de olvidar que el mapa permite y facilita y la VP ejecuta y gestiona.

No obstante, debe hacerse hincapié en que el mapa de variabilidad espacial del viñedo presenta ciertas limitaciones y para potenciar sus ventajas, es necesario comprender la naturaleza y las interrelaciones entre las propiedades de los diferentes factores que explican la variabilidad de cada sector y es muy conveniente vincular estos factores con las propiedades y la calidad de la uva y el vino. Obviamente las características de cada sector son propias y específicas de él y no deben extrapolarse a otras situaciones por más próximas o semejantes que parezcan.
Tabla 1. Actuaciones (gestión o manejo) que permite y facilita el Mapa de Variabilidad del Terroir (MVT).

\begin{tabular}{|c|c|}
\hline Elección del sitio & $\begin{array}{l}\text { Microzonificación, MVT, UHM, } \\
\text { UM }\end{array}$ \\
\hline $\begin{array}{l}\text { Elección del Sistema de } \\
\text { Gestión }\end{array}$ & MC; Manejo Mixto; VP \\
\hline Material vegetal & Variedades, patrones, clones \\
\hline $\begin{array}{l}\text { Conservación de suelos } \\
\text { y aguas }\end{array}$ & $\begin{array}{l}\text { Elección de medidas de pre- } \\
\text { vención de la erosión y de } \\
\text { conservación de suelos y aguas y } \\
\text { detectar el lugar de su aplicación }\end{array}$ \\
\hline Preparación del suelo & $\begin{array}{l}\text { Elección adecuada de las labores } \\
\text { previas a la plantación y detección } \\
\text { del lugar de actuación: desfonde, } \\
\text { subsolado, labor profunda, topo, } \\
\text { volteo, chisel, vertedera,... }\end{array}$ \\
\hline Diseño de riego & $\begin{array}{l}\text { Optimización y localización de la } \\
\text { alimentación hídrica y la gestión } \\
\text { del agua: tipo/forma; diseño; do- } \\
\text { sis, caudal, frecuencia; Sectores }\end{array}$ \\
\hline Pre-plantación & $\begin{array}{l}\text { Enmiendas orgánicas y minerales; } \\
\text { Fertilización/abonado de fondo }\end{array}$ \\
\hline $\begin{array}{l}\text { Material vegetal y } \\
\text { diseño de la formación }\end{array}$ & $\begin{array}{l}\text { Selección y localización de por- } \\
\text { tainjertos y variedades; } \\
\text { Gestión diferenciada del dosel } \\
\text { vegetal y de la poda en los lugares } \\
\text { precisos; }\end{array}$ \\
\hline Plantación & $\begin{array}{l}\text { Optimización del diseño de la } \\
\text { plantación: geometría; sistema, } \\
\text { tamaño de unidades/módulo; } \\
\text { tipo/modo; UM }\end{array}$ \\
\hline Mantenimiento del suelo & $\begin{array}{l}\text { Optimización y localización del } \\
\text { manejo del suelo }\end{array}$ \\
\hline Cubiertas & $\begin{array}{l}\text { Diseño y manejo de cubiertas } \\
\text { específicas para sector: inertes } \\
\text { o vegetales; ciclo vegetativo; } \\
\text { manejo }\end{array}$ \\
\hline Nutrición mineral & $\begin{array}{l}\text { Localización de la aplicación de } \\
\text { los diferentes insumos en función } \\
\text { de las necesidades reales de } \\
\text { cada sector: enmiendas; fertil- } \\
\text { ización, fertirrigación; manten- } \\
\text { imiento/restitución }\end{array}$ \\
\hline Tratamientos & $\begin{array}{l}\text { Tratamiento diferencial y local- } \\
\text { ización de plagas y enfermedades }\end{array}$ \\
\hline Maduración & $\begin{array}{l}\text { Seguimiento del ciclo y de la } \\
\text { maduración específico de cada } \\
\text { sector }\end{array}$ \\
\hline Vendimia & $\begin{array}{l}\text { Gestión de la vendimia y elección } \\
\text { del momento adecuado en cada } \\
\text { sector; }\end{array}$ \\
\hline \multirow[t]{2}{*}{$\mathrm{SIG} / G I S$} & $\begin{array}{l}\text { Seguimiento de la plantación: ali- } \\
\text { mentación hídrica, mineral, vigor, } \\
\text { estado sanitario, plagas } \\
\text { Investigación (diseños experimen- } \\
\text { tales) }\end{array}$ \\
\hline & $\begin{array}{l}\text { Control temporal (anual, bian- } \\
\text { ual,...) de los parámetros de } \\
\text { variabilidad }\end{array}$ \\
\hline Trazabilidad & $\begin{array}{l}\text { Seguimiento del producto: } \\
\text { UM - UP - Mercado }\end{array}$ \\
\hline
\end{tabular}

\section{Realización del MVT}

El MVT es la respuesta a la pregunta ¿dónde y cómo efectuar las diferentes actuaciones (manejo)? y 
las unidades que lo conforman, bien definidas, bien caracterizadas y bien cartografiadas (UHM), son la base de las UM que permiten el diseño de una viticultura de tal detalle que sea consecuente con las modernas ideas de trazabilidad suelo-planta-uva-bodega-mercado.

La calidad del MVT se corresponde con la de las fuentes de información y con las técnicas cartográficas utilizadas para su realización, está en la precisión y exactitud requerida por una escala adecuadamente elegida de acuerdo con los objetivos, en la densidad de observaciones y en las técnicas de muestreo del suelo seleccionados y en la importancia y el número de propiedades, variables o parámetros considerados en las descripciones y los análisis y finalmente en un lenguaje internacionalmente admitido que facilite la transferencia de tecnología.

En los párrafos siguientes se describen brevemente los diferentes procesos manejados en la realización del MVT, en una determinada parcela, imprescindible para el diseño de las UM con la siguiente secuencia: objetivos y escala, metodología y procedimiento, muestreo, información y lenguaje y finalmente, interpretación de resultados.

\subsection{Objetivos y escala}

La escala del mapa es importante porque está relacionada con la cantidad y la calidad de la información proporcionada al viticultor. En un mundo en el que las herramientas de trabajo que, como los Sistemas de Información Geográfica (SIG), facilitan enormemente el manejo y la presentación de resultados (ampliaciones y reducciones, diseño, colores...) es necesario utilizar criterios de calidad del mapa fijados con propiedades relacionadas con la escala, tales como el objetivo y el muestreo.

En este sentido, si el promotor del trabajo es la Administración o un organismo privado y los objetivos se relacionan con la planificación y la ordenación del cultivo de la viña en una zona geográfica relativamente amplia, la escala adecuada sería 1:25.000, y en casos especiales podría ser inferior o igual a 1:50.000. Sin embargo, para viticultores y empresas vitícolas cuyos objetivos se relacionan con la gestión y el manejo de la parcela que condiciona actuaciones directas sobre ella (Tabla 1), la escala necesaria sería al menos 1:5.000 y mejor aún, para casos específicos, sería más recomendable trabajar con escalas superiores, por ejemplo, 1:2.500 [4].

\subsection{Metodología para la identificación y separación de sectores}

El problema principal que se plantea es la diferenciación de sectores y su delineación, la obtención de polígonos o recintos, principalmente porque sobre el dibujo resultante se hace el diseño del muestreo (¿dónde y cómo se hacen las observaciones de campo?) y, a partir de él, el MVT. El objetivo principal consiste en que los sectores iguales deben aparecer en el mapa como iguales y los diferentes como diferentes: parece obvio, pero eso es un mapa. Este conjunto de polígonos en el que todavía no hemos identificado o definido, ni suelos, ni terroir, ni UHM, pero si los hemos identificado, lo denominamos "mapa de sectores".

Para conseguir este mapa de sectores se emplean tres tipos de técnicas específicas: la fotointerpretación aérea (FIA), la percepción remota (remote sensing) o teledetección y el método directo (MD).

La FIA consiste en la utilización de la información que procede principalmente de los fotogramas aéreos convencionales para visión estereoscópica. La visibilidad de diferentes elementos en los fotogramas aéreos con visión estereoscópica (básicos como la pendiente..., compuestos como el patrón de drenaje, ... o deducidos como las formas de paisaje, ...) es de gran importancia para la definición de la variabilidad horizontal del suelo, y su tradicional uso en Edafología [5-7] es debido también a que facilita su delineación y por este motivo la FIA es un instrumento metodológico muy efectivo para la realización del mapa de sectores, más aún a escalas medias que a escalas grandes. Sin embargo, fotointerpretación no es foto-adivinación.

La Teledetección es el conjunto de metodologías que permiten el tratamiento de imágenes obtenidas mediante sensores remotos transportados por cualquier medio: satélites, aeronaves o aviones de alta, media o baja altitud, tripulados o no, automóviles o no, ... La información de objetos o fenómenos (el medio natural) se trata a partir de imágenes espectrales de alta resolución (IEAR) obtenidas por tales sensores. La resolución es una medida de la habilidad que posee un sistema sensor para distinguir entre respuestas que son semejantes espectralmente o próximas espacialmente. Existen varios tipos de resolución (espacial, espectral, radiométrica, temporal) que permiten la selección de imágenes adecuadas para un objetivo determinado. Hoy día este método de diferenciación de sectores adquiere gran importancia principalmente por su fácil acceso, bajo coste y porque también aumenta la precisión de los límites entre sectores de diferenciación a escalas grandes [8].

La cartografía obtenida por este método recoge la respuesta obtenida por el sensor de los elementos que hay en la parcela sólo en el preciso momento en el que pasa el vehículo en el que este se ha instalado. Por lo tanto, este mapa representa la respuesta del estado "electromagnético" de una parcela en un momento determinado sin entrar en las causas de su variabilidad. Por este motivo los mapas resultantes carecen de estabilidad y son diferentes no sólo en función de las propiedades del terroir, sino también, -y esta es la diferencia esencialde las propiedades del medio (elementos meteorológicos, estado del suelo, fenología y estado fisiológico de la planta, etc) en el momento de realización del proceso y hasta las diferentes propiedades de la tecnología disponible.

Por lo tanto, también se puede afirmar que teledetección no es tele-adivinación y en el estado actual de nuestros conocimientos no es posible cartografiar el conjunto de las propiedades permanentes del medio y de las causas de su variabilidad únicamente mediante Teledetección. En particular, el suelo no se ve y por ello es imprescindible el uso de otras técnicas, especialmente en los mapas de escalas grandes y muy grandes.

En tales mapas la utilización del MD adquiere mayor relevancia. El MD obtiene la información mediante técnicas cartográficas de muestreo que se aplican frecuentemente en disciplinas relacionadas con el medio natural como la Climatología, la Geología, la Geomorfología, la Edafología [4].

Este método se optimiza con el mejor conocimiento intrínseco de la parcela (diferencias en la pendiente y/o 
en la exposición; existencia de variaciones estructurales como terrazas o bancales, nivelaciones, desagües o drenajes; variabilidad de la vegetación natural y/o de los cultivos, su crecimiento y su vigor; humedad relativa e hidromorfía; salinidad u otros accidentes negativos; respuesta diferencial al manejo y/o paso de maquinaria; etc), pero con técnicas específicas de muestreo, como la malla fija (fixed grid), no es imprescindible.

En conclusión, la variabilidad sólo puede ser considerada correctamente a partir de una cartografía suficientemente detallada en la que aparezcan delineadas todas y cada una de las unidades, agrupadas las que son iguales y separadas las que son diferentes a causa de alguna propiedad de cualquiera de los factores. Por estos motivos parece lo más sensato, como se ha dicho más arriba, utilizar todo el conjunto de las metodologías disponibles, concretamente el MD, la FIA y la teledetección preferentemente a partir de IEAR, de forma coordinada y complementaria y aprovechando las ventajas de todas estas técnicas para la elaboración adecuada del mapa de sectores, base de los MVT o mapas de UHM con la finalidad última de que a corto o medio plazo (futuro), mediante la gestión llevada a cabo con VP, los sectores de la parcela tengan la máxima homogeneidad posible y así también el producto la tenga.

\subsection{Diseño y realización del muestreo}

Una vez que se dispone del dibujo en el que aparece cada recinto con su contenido diferenciador (el mapa de sectores) se procede al diseño del muestreo cuyo punto de partida es la determinación del número de observaciones que hemos de realizar. Generalmente el cálculo inicial se efectúa por unidad de superficie: densidad de observaciones.

La densidad de observaciones relacionada con el mapa de suelos está condicionada por la escala elegida y, junto con el número de determinaciones que se han de realizar en el laboratorio de acuerdo con los objetivos previstos, condicionan su calidad y su coste [4].

El objetivo del muestreo es identificar, calificar, caracterizar y determinar la distribución de todos los tipos de terroir. El componente del terroir de onda más corta, de mayor variación, y que por lo tanto influye más en la variabilidad del terroir, es sin duda el suelo. Por este motivo el objetivo preferente es identificar, calificar, caracterizar y determinar la distribución de todos los tipos de suelo y para ello se realizan dos tipos de observaciones: las calicatas y los sondeos.

Las calicatas se utilizan para identificar, calificar y caracterizar los suelos. La calicata se realiza de forma manual o mecánica, mediante una retroexcavadora, y con unas dimensiones aproximadas de $2 \mathrm{~m}$ de largo, $0.4 \mathrm{~m}$ de ancho y $2 \mathrm{~m}$ de profundidad. En la calicata se aprecia el perfil del suelo y constituye la unidad de muestreo.

Los sondeos se emplean para definir correctamente la distribución de los suelos, corrigiendo sus límites y los errores de identificación. El sondeo se realiza con una barrena o sonda, manual o mecánica y en él no se aprecia el perfil del suelo completamente y, debido a su finalidad, la mayoría de las veces es suficiente con describirlo para su correcta identificación con algún tipo de suelo ya caracterizado en una calicata y sólo en ocasiones particulares es necesario el muestreo y el análisis.

La densidad de observaciones es definida por diferentes metodologías [4]. La resolución de la OIV [9] recomienda para los estudios que nos ocupan una densidad igual a una observación por cada $\mathrm{cm}^{2}$ de mapa lo que significa 4 observaciones/Ha para mapas a escala 1:5.000 y 16 observaciones/Ha para los de escala 1:2.500. En general, el número de calicatas puede suponer un $25 / 35 \%$ del número de observaciones y el de sondeos el resto.

Una vez determinado el número de observaciones se procede a su localización en el mapa de sectores por alguno de los métodos de muestreo disponibles. Estos métodos de muestreo pretenden una distribución de las observaciones previstas de manera que cumplan sus objetivos de la forma menos costosa posible. Hay muestreos en malla fija (fixed grid), líneas de muestreo (simple line), bandas de muestreo (simple strip), sitios o puntos de muestreo (simple spot) y áreas de muestreo (simple área). Lo más frecuente es que se utilice una combinación de varios de estos métodos en función de las peculiaridades de la zona y el objetivo del estudio.

Para los estudios detallados y muy detallados a los que nos referimos, y que pretenden caracterizar cada sector y detectar la variabilidad dentro de él (intersector), se suele utilizar el muestreo en malla fija (fixed grid) o sus variantes dirigidas. En la malla fija las observaciones se sitúan a lo largo de líneas imaginarias, paralelas entre sí, con igual (cuadrado) o diferente (rectángulo) separación que la existente entre las líneas. El error en los límites entre sectores (precisión) queda definido por la mitad de la distancia entre líneas y de la distancia a lo largo de ellas. El muestreo en malla fija es de coste variable (en función de la amplitud de la malla), pero ciertamente costoso, aunque, sin duda, no caro y si el más favorable.

En situaciones, en las que la disponibilidad de recursos es limitada, se puede optar por un muestreo dirigido a sectores definidos por la intersección de los mapas FIA y de teledetección con la malla fija de lado tan amplio como lo permita el número de observaciones calculado. La malla puede ser variable de forma que sea más densa en sectores en los que el conocimiento de la parcela permita inferir que son más heterogéneos y más amplia en aquellos considerados más homogéneos.

Con demasiada frecuencia se encuentran manuales, y tutoriales en la red, que explican dónde y cómo tomar la muestra que incluso califican de "representativa". Las indicaciones sobre la localización se suelen referir al lugar de forma ambigua (bajo o alto, ladera o llanura, encharcable o no, pedregoso o no, etc) y son, como poco, confusas, sólo consideran la ¿variabilidad? de una de las características del sector $\mathrm{y}$, sobre todo, no permiten delimitarlo. De la misma manera, cuando describen la toma de muestras, se refieren frecuentemente a una muestra superficial que además procede de la mezcla de varias muestras obtenidas siguiendo un itinerario más o menos tortuoso. Como parece obvio, después de las consideraciones realizadas en los párrafos anteriores, esto es, cuando menos, incorrecto: las plantas no toman un determinado elemento por su valor medio, si en un sector es deficiente pueden presentar carencias y si es excesivo, toxicidades. 


\subsection{Descripción de las observaciones}

Una vez localizadas las observaciones en el mapa de sectores se procede a su apertura, descripción y muestreo en el campo.

Como ya se ha dicho el suelo es tridimensional y se estudia a partir de su perfil, que está constituido por una o varias capas llamadas horizontes. El conjunto de los horizontes A y B se denomina solum y es un buen índice para valorar la profundidad efectiva -en la que se desarrolla la mayor parte del sistema radicular de la planta-, de ahí la importancia en su alimentación hídrica y nutricional.

En el perfil es imprescindible describir todos los horizontes, muestrear cada uno de ellos y analizar cada muestra independientemente (obviamente sin mezclarlas). Esto es importante porque cada horizonte tiene sus propiedades y no es correcto referirse, por ejemplo, a un suelo arcilloso o arenoso, sino a un horizonte arcilloso o arenoso de un suelo determinado; por ejemplo, en los viñedos es posible que el horizonte A sea francoarenoso, el B arcilloso y el C francolimoso ¿cuál es el adjetivo que debemos aplicar al suelo? Esto incrementa sensiblemente el número de muestras que se han de realizar.

Una vez abierta la calicata (o el sondeo) en determinado sector de la parcela, se procede a su estudio. Este estudio comienza con la apreciación y descripción del entorno exterior (Litología, Geomorfología, Vegetación natural y usos) y continúa con la visualización del frente soleado del interior. En esta última fase se comienza con la diferenciación de los horizontes y la descripción de sus propiedades (Tabla 2) y termina con el muestreo de cada uno de ellos: tantas muestras como horizontes ( $\sin$ mezclarlas) envasadas y convenientemente etiquetadas para su envío al laboratorio donde se realizarán las determinaciones necesarias (\& 5.5).

Las propiedades descritas en el campo [12] y las determinaciones realizadas en el laboratorio [13] son las que aportan la información precisa, disponible para el viticultor $\mathrm{y}$, por lo tanto, también establecen la calidad del estudio, por supuesto, su número afecta al presupuesto.

Con demasiada frecuencia determinadas consultoras y laboratorios basan sus servicios de asesoría al viticultor en análisis realizados a una determinada muestra de suelo y en el mejor de los casos añaden otra del "subsuelo", y obvian todas las propiedades específicas del perfil y las morfológicas de sus horizontes; así se pierde la mayor parte de la información. Lo correcto es disponer de una descripción completa realizada a partir de una calicata.

Las propiedades morfológicas y físicas del suelo permiten poner un especial interés en los problemas e impedimentos naturales que se plantean en la elección del sitio y en la planificación de las actuaciones (manejo) tanto previas a la plantación, como durante su desarrollo, lo que permite disminuir, o en su caso eliminar, las dificultades para el desarrollo radicular de la planta y su alimentación hídrica. El ejemplo de la densidad aparente (Da) de cada horizonte es paradigmático. Es imprescindible disponer del valor de la Da en condiciones de campo porque permite ajustar y valorar la importancia de la profundidad efectiva y el peso del suelo natural y ahorrar muchos recursos (frecuentemente más del 60\%) relacionados con la fertilización, el agua de riego, etc.
Tabla 2. Propiedades morfológicas a caracterizar para el Mapa de Variabilidad del Terroir (MVT).

\begin{tabular}{|c|c|}
\hline PROPIEDADES & $\begin{array}{l}\text { FINALIDAD Y } \\
\text { UTILIZACIÓN }\end{array}$ \\
\hline Clasificación/Serie $^{1}$ & \multirow{2}{*}{$\begin{array}{l}\text { Comunicación y transferencia } \\
\text { de tecnología }\end{array}$} \\
\hline Clasificación/Horizontes $^{1}$ & \\
\hline Colores y moteados ${ }^{2}$ & $\begin{array}{l}\text { Detección de hidromorfia, en- } \\
\text { charcamientos y calidad }\end{array}$ \\
\hline Concreciones y costras 3 & Limitaciones, manejo \\
\hline Compacidad/porosidad $^{3}$ & Limitaciones, manejo \\
\hline Consistencia $^{3}$ & Laboreo, manejo \\
\hline Densidad aparente $\mathrm{e}^{3,4}$ & $\begin{array}{l}\text { En campo: relaciones } \\
\text { volumen-suelo; peso del } \\
\text { suelo }\end{array}$ \\
\hline Drenaje/desagüe $^{3}$ & $\begin{array}{l}\text { Limitaciones, manejo y } \\
\text { calidad }\end{array}$ \\
\hline Elementos gruesos $^{4}$ & Laboreo, mulching y calidad \\
\hline Escorrentía $^{3,4}$ & Erosión y balance de humedad \\
\hline Espesor de horizonte ${ }^{3}$ & Vigor, fertilidad y calidad \\
\hline Estructura $^{3}$ & Limitaciones y calidad \\
\hline Limitaciones y panes $^{3}$ & Limitaciones y manejo \\
\hline Límites entre horizontes $^{3}$ & $\begin{array}{l}\text { Limitaciones, manejo y } \\
\text { calidad }\end{array}$ \\
\hline Pedregosidad $^{3}$ & Limitaciones, manejo \\
\hline Perfil cultural $^{5}$ & $\begin{array}{l}\text { Detección de posibles } \\
\text { obstáculos para las raíces; } \\
\text { estimación del peso-volumen } \\
\text { útil }\end{array}$ \\
\hline Permeabilidad, CH; infiltración ${ }^{3}$ & $\begin{array}{l}\text { En campo: relaciones suelo } \\
\text { agua, riego }\end{array}$ \\
\hline Profundidad (efectiva) ${ }^{3}$ & $\begin{array}{l}>80 / 85 \mathrm{~cm} . \text { En el campo: } \\
\text { volumen de suelo útil } \\
\text { explorado por las raíces; peso } \\
\text { del suelo (Muy importante) }\end{array}$ \\
\hline Raíces $^{3}$ & Pe, limitaciones, manejo \\
\hline Rocosidad $^{3}$ & Limitaciones, manejo \\
\hline${ }^{1}[10] ;{ }^{2}[11] ;$ & \\
\hline
\end{tabular}

\subsection{Análisis de las muestras: elección y definición de las determinaciones analíticas}

Es frecuente que los responsables de la gestión del viñedo pregunten por la realización al "suelo" de un "análisis completo". Ya se ha señalado que las determinaciones se realizan a cada muestra de cada horizonte y no al "suelo". Cuando esto queda aclarado, la respuesta fácil es dar una referencia en la que figuren la totalidad de las determinaciones que se pueden realizar a una muestra -ver por ejemplo, [13]-, sin embargo esto encarece mucho cualquier estudio $\mathrm{y}$, desde luego, no es lo recomendable.

¿Qué análisis (determinaciones) hay que hacer? Optimizar la respuesta es complicado. Las determinaciones analíticas que se han de realizar a una muestra dependen de muchos factores, unos relacionados con el medio y con la heterogeneidad del sector, otros con la escala, otros con el objetivo y la finalidad del estudio y otros con el tipo de suelo y las características que hemos observado previamente en la descripción morfológica del perfil y en concreto de la propia muestra.

$\mathrm{Ni}$ a todas las muestras se le realizan las mismas determinaciones, ni todas se analizan completamente ¿para qué se necesitan, por ejemplo, los datos de materia 
Tabla 3. Propiedades físicas, químicas, físico-químicas y mineralógicas a determinar [13] para el Mapa de Variabilidad del Terroir (MVT).

\begin{tabular}{|c|c|c|}
\hline \multicolumn{2}{|c|}{ PROPIEDADES } & $\begin{array}{l}\text { FINALIDAD Y } \\
\text { UTILIZACIÓN }\end{array}$ \\
\hline \multicolumn{2}{|c|}{$\begin{array}{l}\text { Conductividad Eléctrica, } \\
\mathrm{CE}\end{array}$} & $\begin{array}{lll}\text { Salinidad, valoración inicial } \\
\text { del riesgo }\end{array}$ \\
\hline \multicolumn{2}{|l|}{$\mathrm{pH}(1: 2,5)$} & $\begin{array}{l}\text { Índice del estado general del } \\
\text { suelo }\end{array}$ \\
\hline \multicolumn{2}{|c|}{ Elementos gruesos } & $\begin{array}{l}\text { Granulometría de la fracción } \\
\text { mineral, propiedades hídricas, }\end{array}$ \\
\hline \multicolumn{2}{|l|}{ Textura USDA } & $\begin{array}{l}\text { minerales..., límite abrupto, } \\
\text { contrastes, etc. }\end{array}$ \\
\hline \multicolumn{2}{|c|}{ Materia orgánica, MO } & Contenido en MO del suelo \\
\hline \multicolumn{2}{|c|}{$\mathrm{N}$} & $\begin{array}{l}\text { Contenido en nitrógeno total } \\
\text { del suelo }\end{array}$ \\
\hline \multicolumn{2}{|l|}{ Relación C/N } & $\begin{array}{l}\begin{array}{l}\text { Evolución de la } \\
\text { orgánica }\end{array} \\
\end{array}$ \\
\hline \multicolumn{2}{|l|}{$\mathrm{P}$} & Fósforo \\
\hline \multicolumn{2}{|l|}{$\mathrm{N} / \mathrm{P}$} & Antagonismo entre el $\mathrm{N}$ y el $\mathrm{P}$ \\
\hline \multicolumn{2}{|l|}{$\mathrm{CIC}$} & $\begin{array}{l}\text { Capacidad de Intercambio } \\
\text { Catiónico: Fertilidad potencial }\end{array}$ \\
\hline \multicolumn{2}{|c|}{ Bases de cambio } & $\begin{array}{l}\text { Suma de bases, SB; Ca, Mg, } \\
\text { Na y K }\end{array}$ \\
\hline \multicolumn{2}{|c|}{ Acidez de cambio; Al } & Suelos ácidos, toxicidad del Al \\
\hline Relaciones & $\begin{array}{l}\text { V; } \\
\text { PCI, }\end{array}$ & $\begin{array}{l}\text { \% de saturación: } \\
V=100 * \text { SB/CIC } \\
\text { Fertilidad actual. }\end{array}$ \\
\hline $\begin{array}{l}\text { en el } \\
\text { Complejo }\end{array}$ & \multirow{3}{*}{$\begin{array}{l}\mathrm{PMI} ; \\
\mathrm{PKI} ; \\
\mathrm{PSI} ; \\
\mathrm{Ca} / \mathrm{Mg} ; \\
\mathrm{K} / \mathrm{Mg}\end{array}$} & $\%$ de $\mathrm{Ca}, \mathrm{Mg}$ y K sobre la CIC \\
\hline \multirow{2}{*}{ de Cambio } & & $\begin{array}{l}\% \text { Na sobre } \\
\text { Alcalinidad }\end{array}$ \\
\hline & & Antagonismos \\
\hline \multicolumn{2}{|c|}{$\begin{array}{l}\text { Caliza total y activa, CA; } \\
\mathrm{Fe}\end{array}$} & $\begin{array}{l}\mathrm{Ca} \text {, agua, clorosis, patrones; } \\
\text { Índice de Poder Clorosante } \\
\left(\mathrm{IPC}=\mathrm{CA} / 10_{*}^{4} \mathrm{Fe}^{2}\right)\end{array}$ \\
\hline \multirow{4}{*}{$\begin{array}{l}\text { Extracto de } \\
\text { Saturación } \\
\mathrm{CE}>0,2 \mathrm{dS} / \mathrm{m}\end{array}$} & Aniones & Salinidad y tipología de sales. \\
\hline & Cationes & Problemas limitantes y riesgos \\
\hline & $\begin{array}{l}\text { CEe; } \\
\text { pHe }\end{array}$ & $\begin{array}{l}\text { de alcalinización (SAR) y de } \\
\text { toxicidad de la planta, Cálculo } \\
\text { de lavado }\end{array}$ \\
\hline & Hs & $\begin{array}{l}\text { Humedad de saturación, } \\
\text { porosidad }\end{array}$ \\
\hline \multicolumn{2}{|c|}{ Oligoelementos } & $\mathrm{Cu}, \mathrm{Zn}, \mathrm{Mn}, \mathrm{B}, \mathrm{Fe}, \mathrm{Pb}, \ldots$ \\
\hline \multicolumn{2}{|c|}{ Constantes de Humedad } & $\begin{array}{l}\mathrm{pF} \text { a } 33 \mathrm{kpa} \text { ya } 1500 \mathrm{kpa} ; \\
\text { régimen de humedad y riego }\end{array}$ \\
\hline \multicolumn{2}{|c|}{ Análisis especiales } & $\begin{array}{l}\text { Mineralogía de arenas y limos; } \\
\text { correlación }\end{array}$ \\
\hline
\end{tabular}

orgánica de un horizonte situado a más de $180 \mathrm{~cm}$ de profundidad? La casuística es muy grande y es difícil dar recomendaciones generales, pero la mayoría de las veces es suficiente pensar que cada cosa que se haga ha de servir para algo y que es innecesario utilizar recursos inútilmente.

En la Tabla 3 se incluye una relación de las determinaciones analíticas a realizar en el laboratorio con la finalidad de caracterizar correctamente el suelo dentro de la perspectiva del MVT e incluye algunas ideas generales sobre su utilización para la producción de vinos de calidad.

\section{6. ¿Qué actuaciones son necesarias? Importancia del lenguaje e Interpretación de resultados}

Una vez definido el mapa de sectores, realizado el muestreo y analizadas las muestras, se ha de llevar a cabo una operación importante que se denomina "correlación" y que pretende dar contenido a este mapa en términos de tipo de suelo de forma que podamos crear las UHM.

Para realizar esta correlación es necesario emplear un lenguaje que sea capaz de diferenciar todos los suelos de cada sector en función de las propiedades cuya variabilidad afecte al cultivo de referencia, a la vid. Es posible que esto pueda hacerse de forma personal, creativa y original por parte de cada operador pero hay dos aspectos importantes que hacen que esto no sea ni correcto, ni adecuado, ni útil: el lenguaje debe ser completo y generalizado.

Completo significa que estén previstos todos los suelos, generalizado que lo use la mayor parte de los técnicos (y científicos) para hacer posible la transferencia de tecnología, es decir, el aprovechamiento de la investigación y las experiencias realizadas en suelos de igual nombre, iguales.

Estas dos condiciones sólo las cumple una sistemática, la Soil Taxónomy [4,10,16] que en su categoría más baja (la Serie) considera más de 23.000 tipos de suelo. Otras clasificaciones o leyendas, como la de FAO [17] o las categorías altas de la propia Soil Taxonomy, están pensadas para escalas pequeñas o muy pequeñas [18] y no sirven en absoluto a nuestro cometido. Se podrá decir que ni el viticultor, ni el gestor de la explotación vitivinícola conocen este lenguaje, y es posible que sea cierto, tampoco el ganadero ha de conocer necesariamente el lenguaje del veterinario, incluso a veces puede ser hasta contraproducente.

Una vez clasificado el suelo al nivel más bajo de más detalle-, realizada la correlación entre sectores y tipos de suelo y creadas las series se obtienen las UHM, en definitiva, el mapa de UHM o mapa de zonificación o MVT. Con este mapa se puede interpretar el conjunto de elementos y propiedades de las que se dispone de información y asignar los resultados a una determinada UHM dentro de cada UM. Esta operación (la interpretación de resultados) sólo tiene sentido si el resultado se asigna a una determinada zona geográfica de la UM. Como es obvio para hacer esta asignación se han de diseñar las UM. Este diseño depende de múltiples factores, sobre todo de la elección del tipo de gestión (MC o VP), del objetivo y del tamaño de la UP, y no tiene una solución única.

El objetivo para obtener la mejor opción es la optimización de la calidad del producto, lo que implica que algunas UHM, sin posibilidad de ser mejoradas, deben ser rechazadas. En la Tabla 4 se incluyen algunas consideraciones sobre la relación entre las UM (Viticultura) y las UP (Enología) y la calidad del producto considerando criterios de especificidad, trazabilidad y rentabilidad.

Es de gran importancia, hacer una referencia a los métodos analíticos utilizados. La mejor recomendación al respecto es tratar de utilizar métodos que estén convenientemente calibrados con relación al tipo de suelo, al cultivo - portainjerto y variedad- y al objetivo: producción de vinos de calidad y, si existen problemas para 
Tabla 4. Relación entre las UM (Viticultura) y las UP (Enología) y la calidad del producto.

\begin{tabular}{|c|c|}
\hline $\begin{array}{l}\text { UNIDADES DE } \\
\text { MANEJO (UM) }\end{array}$ & $\begin{array}{c}\text { UNIDADES DE } \\
\text { PRODUCCIÓN (UP) }\end{array}$ \\
\hline \multicolumn{2}{|c|}{ Manejo Convencional (MC): sin demasiadas posibilidades } \\
\hline $\begin{array}{l}\text { - La superficie de cada UM } \\
\text { es variable en la parcela } \\
\text { y sólo está condicionada } \\
\text { por la mecanización. La } \\
\text { geometría del reparto de } \\
\text { las UM es de tipo puzle. } \\
\text { - Las diferentes UHM } \\
\text { pueden estar constituidas } \\
\text { por suelos disímiles } \\
\text { - La plantación es igual en } \\
\text { toda la UM } \\
\text { - El manejo en campo de la } \\
\text { UM es sencillo } \\
\text { - Se realizan las mismas } \\
\text { actuaciones en todas la } \\
\text { UHM de las UM } \\
\text { - Las actuaciones son } \\
\text { definidas por el tipo de } \\
\text { suelo dominante y/o } \\
\text { limitante }\end{array}$ & $\begin{array}{l}\text { - Pueden ser una o } \\
\text { varias UP por cada } \\
\text { UM } \\
\text { - La capacidad de la } \\
\text { UP es independiente } \\
\text { de la superficie y de la } \\
\text { producción de UM } \\
\text { - En la vendimia se } \\
\text { recoge un producto } \\
\text { heterogéneo que se } \\
\text { elabora en la UP } \\
\text { - Baja calidad del pro- } \\
\text { ducto } \\
\text { - El manejo en bodega } \\
\text { es complejo } \\
\text { - No permite trazabili- } \\
\text { dad }\end{array}$ \\
\hline \multicolumn{2}{|c|}{ Viticultura de Precisión (VP): objetivo simple (cada añada) } \\
\hline $\begin{array}{l}\text { - La superficie de cada UM } \\
\text { es variable en la parcela } \\
\text { y está determinada por la } \\
\text { capacidad del conjunto de } \\
\text { las UP y de la superficie de } \\
\text { la UHM mayor y de la UP } \\
\text { mayor } \\
\text { - La plantación es diferen- } \\
\text { cial en la UM según cada } \\
\text { UHM } \\
\text { - El manejo en campo es } \\
\text { complejo, sobre todo de la } \\
\text { plantación } \\
\text { - Se realizan diferentes ac- } \\
\text { tuaciones a cada una de las } \\
\text { UHM de la UM } \\
\text { - Las actuaciones son } \\
\text { definidas por cada tipo de } \\
\text { suelo de cada UHM }\end{array}$ & $\begin{array}{l}\text { - Varias UP: una por } \\
\text { cada UHM de la UM } \\
\text { - Capacidad de cada } \\
\text { UP relacionada con la } \\
\text { superficie de cada } \\
\text { UHM de la UM } \\
\text { - En la vendimia se } \\
\text { recoge un producto } \\
\text { homogéneo } \\
\text { - Alta calidad del pro- } \\
\text { ducto } \\
\text { - El manejo en bodega } \\
\text { es convencional } \\
\text { - Permite trazabilidad }\end{array}$ \\
\hline \multicolumn{2}{|c|}{ Viticultura de Precisión (VP): objetivo ambicioso (a futuro) } \\
\hline $\begin{array}{l}\text { - Las UHM, al cabo 5-n } \\
\text { años, están prácticamente } \\
\text { homogeneizadas } \\
\text { - La superficie de cada UM } \\
\text { es constante en toda la } \\
\text { parcela y está determinada } \\
\text { por la capacidad de la UP } \\
\text { - La plantación es igual en } \\
\text { toda la UM } \\
\text { - El manejo en campo es } \\
\text { sencillo } \\
\text { - Todas las actuaciones son } \\
\text { diferenciales en toda la } \\
\text { UM } \\
\text { - Las actuaciones definidas } \\
\text { por cada tipo de suelo de } \\
\text { cada UHM son cada vez } \\
\text { más parecidas }\end{array}$ & $\begin{array}{l}\text { - Hay una UP para cada } \\
\text { UM } \\
\text { - La capacidad de cada } \\
\text { UP está relacionada } \\
\text { con la superficie de la } \\
\text { UM } \\
\text { - En la vendimia se } \\
\text { recoge un producto } \\
\text { homogéneo } \\
\text { - Alta calidad del pro- } \\
\text { ducto } \\
\text { - El manejo en bodega } \\
\text { es convencional } \\
\text { - Permite trazabilidad }\end{array}$ \\
\hline
\end{tabular}

acceder a esta información, lo mejor es dejarse aconsejar por los responsables de los laboratorios con experiencia. El desvío de los resultados obtenidos en el muestreo respecto a los valores de referencia de todas las propiedades debe ser corregido en cada sector y en el caso de que esto no sea posible la UHM debe ser rechazada.

En este apartado, aunque no referido al suelo, pero sí muy relacionado con él y sobre todo con su uso, es necesario dejar una nota suficientemente explícita respecto al agua de riego: es imprescindible tener información detallada sobre ella, tomar correctamente la muestra y analizarla convenientemente. Las relaciones físicoquímicas agua-suelo deben preverse y, en su caso prevenir inconvenientes, en el informe de suelos, en caso contrario éste sería incompleto, con graves perjuicios para el futuro de la plantación.

A partir de la correcta interpretación de resultados se diseña el plan de actuaciones para antes de la plantación, para el momento de la plantación y para su vida útil. En este sentido, no sólo se ha de fijar el qué y el dónde, sino también el cómo, el cuánto y el cuándo y definir los controles necesarios.

Por ejemplo, para satisfacer las necesidades nutricionales de la viña es necesario, no sólo que ponga a su disposición los elementos necesarios, sino también que los encuentre en el momento oportuno. Esto ha de conseguirse mediante un plan de abonado adecuado en el que el suelo toma un papel protagonista como almacén o despensa; el correcto desarrollo fisiológico de la planta, en sentido amplio, constituye la garantía de acceso a este almacén a través del sistema radicular de la viña. Este plan de abonado debe tener en cuenta esto y además solucionar los problemas que surjan durante la vida de la plantación, respondiendo a las principales cuestiones que el viticultor se plantea: ¿qué fertilizante? (tipo, composición, formulación), ¿cuánto? (dosis de fertilizante y/o enmienda), ¿cuándo? (momento de la realización del abonado, calendario), ¿cómo? (extensión, localización, mecanización) y ¿dónde? (en cada una de UHM de la UM correspondiente). Las respuestas a las cuestiones no tratadas hasta ahora están fuera del ámbito de este trabajo principalmente por problemas de espacio.

Durante la vida de la plantación, su situación evoluciona de tal forma que se crea una variabilidad importante dentro de cada una de las UM de manera que es necesario corregir los posibles desequilibrios generados. Son por ello necesarios análisis de control al menos cada 4/5 años que nos permitan volver a la situación de equilibrio. Estos controles tienen que ver principalmente con el suelo, la planta y sus relaciones.

En cuanto a la planta son útiles las directrices provisionales sobre el muestreo de la planta $[19,20]$ y los análisis foliares y/o peciolares en relación con el suelo y los estados fenológicos; y respecto al suelo, son suficientes controles periódicos del estado sanitario y de la nutrición en el horizonte superficial (A), entre 5 y $20 \mathrm{~cm}$ (MO, N, $\mathrm{CE}, \mathrm{pH}$ y $\mathrm{P}$ ) y en el horizonte subsuperficial (generalmente B) de la fertilidad ( $\mathrm{Ca}, \mathrm{Mg}, \mathrm{Na}, \mathrm{K}$ y acidez de cambio).

\section{Conclusiones}

- El Mapa de Variabilidad del Terroir (MVT), Mapa de los Terroir o Mapa de Zonificación del Terroir constituye la cartografía detallada de la parcela y 
permite conocer la delimitación y la caracterización de los diferentes sectores que constituyen su variabilidad.

- El MVT se sitúa como una necesidad entre el Terroir (ambiental) y la gestión del viñedo y está constituido por el mapa de las UHM y el de las UM. Este mapa facilita y optimiza la gestión independiente de cada uno de los sectores de la parcela de manera localizada en función las características propias de cada sector.

- La información utilizada para obtener el MVT se consigue coordinando los tres tipos de técnicas (FIA, teledetección y MD) y aprovechando todas sus ventajas.

- El MD es la forma usual de conseguir el MVT a escalas grandes. Este método realiza el mapa con eficacia y además aporta información sobre las propiedades de cada terroir, lo que permite diagnosticar las causas de la heterogeneidad, la relación con el producto y la posibilidad de influir sobre ellas de forma adecuada.

- Los mapas obtenidos de IEAR (teledetección) aportan información importante, son muy asequibles, facilitan la precisión de los límites y se obtienen más rápidamente que los realizados a partir del MD. Sin embargo, en el estado actual de nuestros conocimientos no es posible cartografiar el conjunto de las propiedades permanentes del medio y de las causas de su variabilidad únicamente mediante Teledetección.

- En todos los casos la calidad del muestreo es fundamental. La escala, la densidad de observaciones, el número de propiedades implicadas en el estudio y el lenguaje determinan la utilidad del MVT y las directrices, a ser posible siguiendo normas internacionales, deben ser garantía de calidad.

- El MVT así obtenido permite al viticultor abordar el objetivo más ambicioso: la optimización y homogeneización del producto. En concreto se trata de que a corto o medio plazo (futuro) y mediante la gestión llevada a cabo con la Viticultura de Precisión la actuación sobre las propiedades del terroir en los diferentes sectores de la parcela se consiga la mayor homogeneidad posible y así también el producto lo sea.

\section{Referencias}

[1] OIV Definición de "terroir" vitivinícola. Res. OIV VITI 333/2010 (2010)

[2] V. Gómez-Miguel Atlas das castas da Península Ibérica: história, terroir, ampelografia. Dinalivro 3, 104-153 (2011)

[3] V. Gómez-Miguel y V. Sotés Delimitación de zonas vitícolas en la DO Ribera del Duero, en la DO Ca Rioja, en la DO Rueda, en la DO Toro, en la DO Bierzo, en la DO Somontano, en la DO Cigales, en la DO Arribes, en la RD Douro y en la DO campo de Borja. ETSIA-ETSIAAB. Universidad Politécnica de Madrid (1990-2016)

[4] USDA Soil Survey Manual. H. No. 18, 437 (1993)

[5] D. M. Carroll, R. Evans y V. C. Bendelow Air photointerpretation for soil mapping Soil Survey 85 (1977)

[6] FAO Aerial photo interpretation in Soil Survey. Soil Bull. 6 (1967)

[7] L. P. White. Aerial Photography and Remote Sensing for Soil Survey. Oxford Univ. Press 104 (1977)

[8] V. Gómez-Miguel, Á. Martínez y ML. GonzálezSanJosé Nuevos Retos y Oportunidades en Viticultura, Enología y Cultura del Vino 1-19 (2016)

[9] OIV Líneas directrices sobre metodologías de zonificación vitivinícola a nivel del suelo y del clima. Res. OIV-VITI 423-2012 (2012)

[10] USDA Key to Soil Taxonomy (2014)

[11] Munsell Munsell soil color charts (2015)

[12] USDA Field book for describing and sampling soils 3.0 (2012)

[13] USDA Soil Survey Laboratory Methods Manual. SSIR 42, v.5.0 (2014)

[14] S. Henin, R. Gras y G. Monnier Le profil Cultural Masson (1969)

[15] A. Reynier. Manuel de viticulture. Tec \&amp- Doc Lavoisier 587 (2016)

[16] USDA National Soils Handbook (2004)

[17] FAO World reference base for soil resources No 106 (2014)

[18] V. Gómez-Miguel y D. Badía The Soils of Spain. Springer, WSBS 24-98 (2016)

[19] OIV The OIV guidelines for sampling in vineyards. (prop. Prel. mod) CI-TECVIT 2014-03 04.WD23 (2015)

[20] R. B. Ferguson y G. H. Hergert Soil sampling for Precisión Agriculture. Univ.Nebraska IANR. EC 00-154, Lincoln (2016) 